\title{
Optimización y evaluación de la Plataforma Virtual Continental para mejorar la gestión de tutoría virtual
}

\author{
Optimization and evaluation of the Continental Virtual Platform to improve virtual tutoring \\ management
}

Miguel Ángel Córdova Solís

Instituto Continental

\section{RESUMEN}

Objetivos: Desarrollar mejoras informáticas y evaluar la Plataforma Académica Virtual Continental (PAVC) basada en Moodle para optimizar la gestión de tutoría virtual en los programas e-Learning que ofrece la Escuela de Postgrado de la Universidad Continental - sede Lima. Métodos: Se realizó mejoras a nivel de software en la plataforma virtual Moodle, luego, por tratarse de una investigación de nivel exploratorio con diseño pre experimental, con dos grupos y post prueba se realizó la aplicación de un instrumento que permitió medir la percepción de los usuarios. Resultados: El número de recordatorios emitidos por el tutor se incrementó de $13 \%$ a $31 \%$, reporte automático de cumplimiento de actividades se incrementó de 6 a 10 informes, disminución del número de deserciones por motivos de comunicación y atención oportuna de 15 a 5 y el incremento de $19 \%$ a $100 \%$ de los alumnos que han participado en la encuesta on-line; respecto a los administrativos inmersos en estos programas el $100 \%$ manifestó su conformidad por el procesamiento automático de los resultados de las encuestas, el 100\% manifestó que al tener en menor tiempo los informes y reportes, estos les permiten actuar oportunamente en la solución de problemas o consultas de los alumnos, derivados por el tutor. Conclusiones: Las mejoras informáticas implementadas han permitido al tutor cumplir un rol más eficiente, de ello las encuestas on-line implementada constituye el principal aporte de la investigación por los resultados obtenidos.

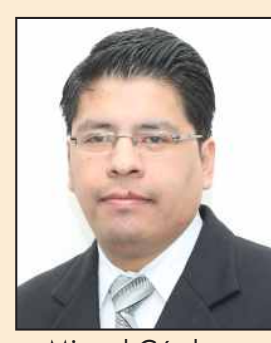

Miguel Córdova

Palabras clave: Plataforma virtual, tutor, eficiencia, aprendizaje electrónico.

1 Ing. de Sistemas, estudios de Maestría en Administración con mención en Informática para la Gestión, Diplomado en Gestión de Cátedras E- Learning, Especialización en Tutoría Virtual. 


\section{ABSTRACT}

Objectives: Develop informatics improvements and evaluate the Continental Virtual Academic Platform (PAVC), based in Moodle to optimize virtual tutoring management in the e-Learning programs that are offered by the postgraduate school of the Continental University - Lima subsidiary. Methods: Improvements took place in a software level in the Virtual Platform Moodle, then, because of it is an exploratory research with pre experimental design and with two groups and post test, an instrument application was realized that allows measure users perception. Results: The remainder number send by the tutor was increased from $13 \%$ to $31 \%$, the automatic report of activities compliance was increased from 6 to 10 informs, decreased the desertions number because of opportune communication and attention from 15 to 5 and the increase from $19 \%$ to $100 \%$ of students that participate on the online survey; respect to the administrative people inmersed on these programs, $100 \%$ manifested their agreement with the automatic processing of survey results, $100 \%$ manifested that having informs and reports on a short time, let them to act opportunely to solve students problems or consults, derived by the tutor. Conclusions: The informatics improvements implemented permitted to the tutor comply a more efficient role, so that the implemented on-line surveys establish the principal investigation contribution because of the obtained results.

Key words: Virtual Platform, tutor, efficiency, electronic learning.

\section{INTRODUCCIÓN}

Se tienen como antecedentes del presente proyecto las investigaciones llevadas a cabo por Andonegui J. (1), Gil P. (2), Borges F. (3), Betancourt A. (4) y Acebal A. (5); quienes demuestran y plantean la importancia y necesidad que las entidades educativas cuenten con la figura de tutor virtual en sus programas a distancia, así como describen las causas y consecuencias de la eficiencia en la labor tutorial en el rendimiento, desempeño y frustración en los participantes.

$\mathrm{Si}$ bien, los programas de e-learning constituyen una importante oportunidad para que los alumnos deseosos de superación y empresas que desean capacitar a su personal logren las competencias tan igual como se lograrían durante las sesiones presenciales; a nivel de postgrado, inclusive, este medio constituye una excelente oportunidad de formación y capacitación, en este escenario, el tutor virtual constituye un actor clave en este proceso por su función de facilitar un espacio dentro de la plataforma virtual donde el alumno y el docente puedan establecer el proceso de enseñanzaaprendizaje, sin embargo muchas actividades como el seguimiento de la participación de los estudiantes de los programas de estudios se realizaba fuera de tiempo, los reportes de notas consolidadas así como el procesamiento de los resultados de las encuestas estudiantiles no estaban automatizados generando ineficiencias en la gestión; ante esta problemática, se planteó la necesidad de realizar mejoras informáticas en la PAVC que influya en la eficiencia de la gestión de Tutoría Virtual de los programas de E-learning que oferta la Escuela de Postgrado de la Universidad Continental.

El objetivo fue desarrollar mejoras informáticas en la Plataforma Académica Virtual Continental (PAVC) para optimizar la gestión de tutoría virtual en los programas eLearning que en este caso se trata del sistema de aprendizaje, mundialmente conocido Moodle.

El primer paso por lo tanto, fue revisar el modelo educativo en la modalidad virtual definido por la Escuela de Post Grado de la Universidad Continental para ubicar el rol y las funciones del tutor virtual, dicho modelo centrado en el estudiante involucra elementos como: manual de 
autoaprendizaje, asistencia virtual de los tutores, sesiones chat, foros de discusión, evaluaciones on line y los ejercicios aplicativos, todos ellos disponibles desde la PAVC.

El Diccionario de la lengua española define al tutor como la persona encargada de orientar a los alumnos de un curso o asignatura (6). Antes que un profesor, en el sentido tradicional de la educación presencial, es un orientador, un apoyo o facilitador de los aprendizajes de los alumnos. La Oficina de Evaluación Tecnológica (7) del Congreso de los Estados Unidos, en un documento de 1989 dejó clara constancia que "la clave del éxito del aprendizaje a distancia es el tutor. Si el tutor es bueno, la tecnología se vuelve casi transparente, al contrario, ninguna tecnología puede superar un pobre proceso tutorial...".

Chaupart, Vitalia y Marín (8) señalan que el tutor, además de los conocimientos específicos de su área y de los principios básicos de la educación a distancia, debe poseer una serie de capacidades, habilidades y competencias; no se pretende reemplazar a este actor, pero si optimizar muchos de sus procesos que son llevados a cabo de manera manual; la cual, muchas veces distrae que se centre en el objetivo principal que es la de guía y facilitador de los alumnos en modalidad a distancia.

Nuestra propuesta de mejoras informáticas desarrolladas en la PAVC influye en la eficacia de la gestión de tutoría virtual de los programas e-Learning que ofrece la Escuela de Postgrado de la UCCl (Lima), en tal sentido es conveniente señalar que la PAVC, es el soporte tele informático de las actividades académicas de la EPGC y se basa en la plataforma educativa MOODLE (Modular Object - Oriented Dynamic Learning Environment), la cual permite la interacción entre el participante con sus compañeros, con el docente y el tutor, es además un medio para presentar sus ejercicios, trabajos o productos programados en cada módulo.

\section{Mejoras informáticas en la PAVC}

La PAVC que dispone de licencia libre ofrece una variada posibilidad de desarrollar e implementar mejoras informáticas; nuestra contribución en la presente investigación se ha basado en desarrollar las siguientes mejoras informáticas:

- Recordatorios automáticos: El módulo de mensajes constituye un sistema de mensajería interna, la cual permite intercambiar mensajes entre los estudiantes, el docente y el tutor. Dicha mensajería no necesita una dirección de correo electrónico ni un cliente de correo, tan sólo el navegador; además que constituye una herramienta que permite al tutor seleccionar destinatarios, un $65 \%$ de estudiante lo emplea, motivo por el cual se ha decidido utilizador como medio para el envío de recordatorios de cumplimiento y entrega de actividades académicos automáticos.

- Reporte automático de cumplimiento de actividades: El informe de participación nos muestra las acciones realizadas por los usuarios, esta es una herramienta desde la cual es posible de generar gráficos dinámicos para un rápido análisis e interpretación de resultados.

- Publicación de notas y promedios: Opción que permite la publicación de las notas y promedios por módulo, según la ponderación definida por el docente.

- Encuestas on-line: Módulo que ha permitido personalizar las preguntas que son remitidas por las Coordinadoras Académicas para medir el nivel de satisfacción a los alumnos; esta herramienta además permite que a partir de las encuestas, generar archivos en MS Excel con los resultados tabulados y procesados.

- Foro de consulta al tutor: Variación del típico foro, que permite al alumno enviar sus consultas para una pronta atención y se ha configurado la opción de enviar una copia del mensaje al email del tutor y del 
estudiante para una mayor prontitud en las respuestas así como realizar el seguimiento de los mensajes.

\section{MATERIAL Y MÉTODOS}

Para el desarrollo de las mejoras, se ha instalado y configurado inicialmente una versión de prueba (demo) Moodle versión 1.9.7 de la PAVC en un servidor web Apache alojado en un hosting de pago con sistema operativo Linux GNU CentOS y gestor de base de datos MySQL, ello para tener una configuración muy similar al PAVC, los clientes han accedido desde computadoras Pentium IV y laptop con el requerimiento que debían tener acceso a Internet y disponer de un navegador actualizado y disponer programas como mínimo Adobe Reader 7 y MS Office 2003.

Las implementaciones se realizaron de manera gradual y la Oficina de Informática de la Organización Educativa Continental apoyó con la instalación del módulo para la encuesta en el servidor del PAVC y la configuración del servidor SMTP (correo electrónico) para el envío automático de mensajes al email.

Debido a que el diseño de la investigación se ha definido como pre experimental no ha existido un grupo de control, la población estuvo conformado por la totalidad de los estudiantes y personal administrativo, a partir del cual se definieron dos muestras claramente diferenciadas:

- Muestra 1: Alumnos participantes de los programas virtuales de la Escuela de Postgrado de la UCCI (Lima): 200 alumnos (mayo 2011)

- Muestra 2: Personal del área académico de la EPGC: Cuatro (4) personas (Dos coordinadoras académicas y dos asistentas académicas).

Al cabo del primer mes de las implementaciones de las mejoras informáticas en la PAVC; para el acopio de datos se empleó entrevistas (muestra no probabilística) y encuestas con escala de Likert (muestra probabilística).

\section{RESULTADOS}

Se ha logrado los siguientes resultados:

- El incremento del número de recordatorios emitidos por el tutor por un mes de $13 \%$ a $31 \%$; reporte automático de cumplimiento de actividades elaborado por el tutor de 6 a 10 informes por mes.

- A mayor prontitud, mayor satisfacción académica, de 34 a 59 alumnos en un mes.

- Disminución del número de deserciones por falta de atención de 15 a 5 .

- Incremento de 19\% a 100\% de los alumnos que han participado en la encuesta on-line.

- El foro de consulta al tutor (50\% de los mensajes enviados de estudiante-tutore) y los mensajes privados (35\%) constituyeron los principales medios de comunicación con los alumnos, en contraste con el empleo del email personal (15\%).

- Monitoreo más rápido y preciso (según reportes de tutoría emitidos durante 1 mes), en cuanto a la prontitud de mensajes de emergencia también se notó un incremento de 2 a 6 (300\%).

- Número de deserciones (motivados por la falta de atención en la PAVC), al realizar la prueba de hipótesis se establece que no existe relación entre ambas variables (Eficiencia en la labor de Tutoría Virtual VS Número de deserciones).

- Se establece relación entre las variables (Herramientas informáticas de gestión académica en la PAVC VS revisión de 
Notas y promedios de alumnos).

- Existe una fuerte relación de X2 =92.3823 a $95 \%$ de confianza, entre las variables (Herramientas informáticas de gestión académica en la PAVC VS Encuestas online) debido a la prueba de hipótesis aplicado.

- En cuanto al Control de notas, se establece mediante la prueba chicuadrada aplicada que no existe relación entre las variables (Herramientas informáticas de gestión tecnológica en la PAVC VS Control de notas).

- Se aprecia que el número de mensajes enviados por los alumnos al foro de consulta al tutor y atendidos inmediatamente (menos a $24 \mathrm{hrs}$ ) es mayor con las mejoras informáticas en la PAVC.

- Control de reclamos de los alumnos en temas técnicos, no se establece una relación entre variables (Herramientas informáticas de gestión tecnológica en la PAVC VS Control de reclamos de los alumnos en temas técnicos).

- La revisión y validación de los recursos y actividades publicados, se muestra un incremento en la mejora en cuanto a la revisión y validación de links publicados por parte del tutor virtual.

Resultados obtenidos de la entrevista (administrativos):

- El 100\% manifestó su conformidad por el procesamiento rápido, confiable y ordenado de los resultados de la encuesta on-line.

- El 100\% manifestó que al tener más rápido los informes y reportes les permite actuar más rápido en solucionar los problemas o consultas de los alumnos, derivados por el tutor.

- Respecto al control de documentos enviados, se establece relación entre las variables (Herramientas informáticas de gestión académica en la PAVC VS Control de documentos enviados (aspectos administrativos)

\section{DISCUSIÓN}

Al disponer de una herramienta automática de envío de recordatorios emitidos por el tutor, el proceso se simplifica y se optimiza, razón que explica el incremento del número de mensajes enviados por el tutor. De igual forma, se explica el incremento del reporte de cumplimiento de actividades elaborado por el tutor y que son remitidos a la asistenta y coordinadoras académicas. Se puede concluir, entonces que las mejoras informáticas implementadas ha permitido al tutor: enviar mayor cantidad de recordatorios a los alumnos debido a la optimización del proceso de envío de mensajes. Asimismo, el incremento del número de reportes e informes que genera el tutor y en muchos casos reporta a las coordinadoras y asistentes académicas, se traduce en un efectivo monitoreo $y$ seguimiento por alumno.

A mayor prontitud en atender las consultas de los estudiantes, mayor satisfacción académica de estos y la disminución del número de deserciones por este motivo, coincide con lo señalado por Borges (3) quien indica que la comunicación es primordial para evitar la frustración de un estudiante en modalidad virtual.

El foro de consulta al tutor y los mensajes privados constituyeron los principales medios de comunicación con los alumnos, en contraste con el empleo del email personal, ello se explica con el concepto de campus Virtual propuesto por Andonegui (1), quien enfatiza la necesidad de incorporar módulos académicos y administrativos dentro de un mismo medio, la cual facilitará un mejor desenvolvimiento por parte del alumno en un entorno virtual.

Se establece relación entre las variables (Herramientas informáticas de gestión académica en la PAVC VS revisión de Notas y 
promedios de alumnos), el incorporar un medio de publicación de notas y promedios finales de una manera ordenada y clara, facilitará la gestión académica de los responsables de esta función como el tutor y las asistentes hacia el alumno.

Existe una fuerte relación entre las variables (Herramientas informáticas de gestión académica en la PAVC VS Encuestas online), ello contrasta con Betancourt (4) quien indica que el estudiante en educación a distancia tiene los mismos derechos que el estudiante presencial y el expresar su opinión mediante las encuestas, es una forma de demostrarlo. Las encuestas on-line implementada constituye quizá el principal aporte de la investigación.

El envío automatizado de mensajes de los foros al email si bien no constituye el principal medio de comunicación, cumple una función informativa y de alerta tanto a los alumnos y al tutor; Gil (2) señala que es necesario proveer de medios internos y externos que permita y asegure la comunicación con el alumno. 


\section{REFERENCIAS BIBLIOGRAFICAS}

1 Andonegi J, Ezeiza A, Fernández F, Garmendia M, Pérez A, Palacios S, Ruiz I Difusión del software libre en la universidad la experiencia piloto Moodle en la Universidad del País Vasco. EDUCAR. 2008; 41 (1): 51-58.

2 Gil P, Pomares J, Candelas FA, Puente ST, Jara C, Corrales JA, et al. Metodología b-learning con Moodle para la enseñanza y evaluación del aprendizaje en las asignaturas de Redes de Computadores. DFISTS, Departmento de Física, Ingeniería de Sistemas y Teoría de la Señal, Escuela Politécnica Superior, Universidad de Alicante; 2008. [Libro virtual]

3 Borges F. La frustración del estudiante en línea. Causas y acciones preventivas. Digithum. 2005; : En: http://www.voc.edu/digithum/7/dt/esp/ borges.pdf

4 Betancourt A. La educación a distancia y la función tutorial. UNESCO, San José Edición Victoria Monturiol; 2008.

5 Acebal A, Tessio N. La tutoría en el campus virtual universitario. Universidad Virtual de Quilmes, Argentina; 2005. [Libro virtual]

6 Real Académica Española. Sitio Web de la Real Academia Española. 2011 ; En: http://buscon.rae.es/drael/SrvltConsulta?TIPO_BUS=3\&LEMA=tutor\%C3\%ADa

7 US Congress - Office of Technology Assessment. Linking for learning: a new course for education. 1989; 10(1): 12-19.

8 Chaupart J, Vitalia M, Marín G. El tutor, el estudiante y su nuevo rol. Libro de Actas del VI Encuentro Internacional de Educación a Distancia. 1998; 2(2): 97-110.

Correo electrónico:

mcordova@continental.edu.pe 